\title{
Lumen
}

Selected Proceedings from the Canadian Society for Eighteenth-Century Studies

\section{Le Créole patriote (1792-1794) : un pont entre deux Révolutions}

\section{Giulia Bonazza}

Volume 35, 2016

URI : https://id.erudit.org/iderudit/1035922ar

DOI : https://doi.org/10.7202/1035922ar

Aller au sommaire du numéro

Éditeur(s)

Canadian Society for Eighteenth-Century Studies / Société canadienne d'étude du dix-huitième siècle

ISSN

1209-3696 (imprimé)

1927-8284 (numérique)

Découvrir la revue

Citer cet article

Bonazza, G. (2016). Le Créole patriote (1792-1794) : un pont entre deux

Révolutions. Lumen, 35, 81-93. https://doi.org/10.7202/1035922ar d'utilisation que vous pouvez consulter en ligne.

https://apropos.erudit.org/fr/usagers/politique-dutilisation/ 


\title{
Le Créole patriote (1792-1794) : un pont entre deux Révolutions
}

\author{
Giulia Bonazza \\ ÉHÉSS / Université Ca' Foscari
}

Milscent et son journal Le Créole patriote s'insèrent dans les dynamiques transatlantiques qui concernent la Révolution française et la Révolution de Saint-Domingue/Haïti. Deux révolutions qui aboutirent à la première abolition de l'esclavage en France, ratifiée par le décret du 16 pluviôse an II (4 février 1794), sous la Convention montagnarde. Le décret représente un des moments politiques les plus significatifs pas seulement parmi les conquêtes juridiques de la période révolutionnaire mais aussi, dans une perspective de longue durée, par rapport aux questions abolitionnistes du XIX ${ }^{\mathrm{e}}$ siècle ${ }^{1}$.

Le biquotidien Le Créole patriote (1792-1794) est rédigé par Claude Louis Michel Milscent De Mussé, dénommé Milscent, un homme libre de couleur et petit colon de Saint-Domingue, qui est venu vivre dans la mère-patrie après l'éclatement de la Révolution française. Milscent est une des figures «marginales» de la Révolution, en incarnant le point de contact entre colonie et métropole. Grâce à sa mobilité et aux échanges épistolaires il contribua à informer l'opinion publique sur les nouvelles de Saint-Domingue. Milscent et son journal incarnent parfaitement - pour reprendre une expression de Lynn Hunt - la fusion entre «action» et «innovation» de la période révolutionnaire².

1. La deuxième abolition de l'esclavage dans les colonies françaises fut sanctionnée par le décret de 1848, dont Victor Schoelcher fut le promoteur. Voir «Des lenteurs parlamentaires aux initiatives individuelles ", dans Nelly Schmidt, Labolition de l'esclavage. Cinq siècles de combats, $X V I^{e}-X X^{e}$ siècle, Paris, Fayard, 2005, p. 199-215.

2. Lynn Hunt, Politics, Culture and Class in the French Revolution, Berkeley, University of California Press, 1984, traduction de Erica Joy Mannucci, La Rivoluzione francese. Politica, cultura, classi sociali, Bologna, Il Mulino, 1989, p. 180. 
L'article s'inscrit chronologiquement à partir de l'insurrection des esclaves - éclatée dans la nuit du 22 août $1791^{3}$ - au décret d'abolition de l'esclavage du 16 pluviôse an $\mathrm{II}^{4}$. La première abolition de l'esclavage met en évidence plusieurs problématiques concernant les liens qui unissent la mère patrie et la colonie. La première problématique concerne la contribution réelle du courant antiesclavagiste de la Révolution française comme moteur du soulèvement des esclaves et comme fondement théorique du décret abolitionniste ${ }^{5}$. La deuxième porte sur la dimension globale de la Révolution de Saint-Domingue: le soulèvement des esclaves qui est - au début - un événement de portée locale, s'insère après dans des dynamiques atlantiques entre la France et les puissances de la contre-révolution ${ }^{6}$.

Penser la Révolution de Saint-Domingue comme un chapitre de la Révolution française est erroné du point de vue méthodologique puisque dans la colonie française éclate une révolution spécifique. En même temps il est fondamental de reconnaitre un pont entre les deux révolutions parce qu'elles ont en commun un même rythme et un même arc chronologique:

Il faut bien qu'on le comprenne; il n'y a pas de «Révolution française » dans les colonies françaises. Il y a dans chaque colonie française une révolution spécifique, née à l'occasion de la Révolution française, liée à elle, mais se déroulant selon ses lois propres et avec ses objectifs particuliers. Toutefois un point commun entre les deux phénomènes est le rythme?.

3. Sur l'insurrection des esclaves à Saint-Domingue, voir Frédéric Régent, $L a$ France et ses esclaves. De la colonisation aux abolitions (1620-1848), Paris, Grasset, 2007, p. 236-237; Laënnec Hurbon (éd.), L'insurrection des esclaves de Saint-Domingue (22-23 août 1791), Actes de la table ronde internationale de Porte-au-Prince (8 au 10 décembre 1997), Paris, Karthala, 2000.

4. Florence Gauthier (éd.), Périssent les colonies plutôt qu'un principe! Contributions à l'étude de l'abolition de l'esclavage, 1789-1804, Paris, Société des Études Robespierristes, 2002, p. 14.

5. Jean-Pierre Le Glaunec, Larmée indigène. La défaite de Napoléon en Haïti, Montréal, Lux, 2014, p. 46.

6. Sur l'antiesclavagisme transnational et pas seulement français, Alessandro Tuccillo, Il commercio infame: antischiavismo e diritti dell'uomo nel Settecento italiano, Naples, Clio Press, 2013, p. 30-42.

7. Aimé Césaire, Toussaint Louverture. La Révolution française et le problème colonial, Paris, Présence Africaine, 1981 [1962], p. 24; Cf. Fabien Marius-Hatchi, «La Révolution caribéenne comme ultime rempart du droit naturel. Déchoukaj et coup 
Par conséquent reconnaître la contribution de la Révolution éclatée dans la mère patrie sur l'éclatement de la Révolution coloniale et considérer la révolte des esclaves comme accélératrice des événements dans la métropole n'est pas contradictoire. D’un côté, on a l'approche éclairante de l'historien D.P. Geggus: «the colonial revolution as an autonomous force that helped radicalize the French Revolution rather than being merely a reflection of it ${ }^{8} »$. D'un autre côté, l'énergie jaillie du processus révolutionnaire français a démonté l'immuable société coloniale pour créer un climat politique favorable à l'éclatement de l'insurrection des esclaves?.

Du point de vue politique, la révolte des esclaves accélère, au-delà des principes révolutionnaires, la prise de décision de la mère patrie sur le problème de l'esclavage. La Révolution de Saint-Domingue acquiert officiellement à partir du février 1793 une dimension internationale : l'occupation de la Reine des Antilles devient un objectif pour les puissances de la contre-révolution, l'Espagne et l'Angleterre. Les liens métropole-colonie et colonie-métropole manifestent que les deux processus révolutionnaires ne sont pas totalement indépendants ${ }^{10}$.

Du point de vue historiographique, Milscent a été découvert grâce aux études de Yves Bénot. Il existe aussi un article de Jean-Daniel Piquet et plus récemment il y a une reconstruction détaillée de son parcours et de ses œuvres dans l'article «Milscent créole historien de la Révolution de Saint-Domingue 1790-1794» de Sophie Piollet, Nathalie Piquionne et Delphine Roux ${ }^{11}$. Yves Bénot a découvert la source - Le

d'arrêt thermidorien aux îles du vent 1794-1795 ", dans Florence Gauthier (éd.), Périssent les colonies, op. cit., p. 68.

8. David Patrick Geggus, Haitian Revolutionary Studies, Bloomington, Indiana University Press, 2002, p. 167 .

9. Sur l'interprétation historiographique pour laquelle la Révolution française a eu un impact fondamental sur la révolution haïtienne, voir Jürgen Osterhammel, Die Verwandlung der Welt. Eine Geschichte des 19. Jahrhunderts, traduction en anglais, The Transformation of the World. A Global History of the Nineteenth Century, Princeton/Oxford, Princeton University Press, 2014 (2009), p. 528-529.

10. Déclaration de guerre à l'Angleterre par la Convention girondine, Florence Gauthier, Triomphe et mort du droit naturel en Révolution 1789-1795-1802, Paris, PUF, 1992, p. 213.

11. Yves Bénot, La Révolution française et la fin des colonies, Paris, La Découverte, 1987 , p. 54, 83, 84, 120, 166, 167, 168, 172, 175, 185, 196, 217 et 241; Jean-Daniel Piquet «Le Créole Patriote, apôtre de l'insurrection de St-Domingue », Annales historiques de la Révolution française, 293-294, 1993, p. 519-521; Laënnec Hurbon (éd.), 
Créole patriote - mais il n’a pas retrouvé les numéros conservés à la Bibliothèque historique de la ville de Paris (BHVP); Jean-Daniel Piquet est allé plus loin, mais dans l'article, il a analysé seulement les onze parties du journal qui portent le titre Colonies et qui vont du $n^{\circ} 276$ au $\mathrm{n}^{\mathrm{o}} 300^{12}$. Larticle de Sophie Piollet, Nathalie Piquionne et Delphine Roux apporte une contribution importante, en contextualisant le journal dans le débat théorique antiesclavagiste et en reconstruisant la biographie de Milscent, mais il ne se focalise pas sur la dimension internationale et en particulier sur la dimension transatlantique de la question et il ne tient pas compte de tous les numéros du biquotidien ${ }^{13}$.

Mon objectif est de montrer l'interconnexion entre les deux révolutions et comment Milscent en est le point de conjonction. En outre je voudrais chercher à contextualiser le biquotidien non seulement à l'intérieur de la pensée antiesclavagiste, mais aussi dans les événements spécifiques qui ont marqué la période révolutionnaire comme le rapport métropole-colonie. La contribution antiesclavagiste de Milscent est fondamentale dans une double perspective. La première concerne son rôle d'informateur privilégié des événements dominicains au service de l'opinion publique de la métropole. La deuxième concerne la factibilité faisabilité des solutions que Milscent propose pour résoudre le problème de l'esclavage en dépassant les limites du débat théorique. Grâce au journal, il est possible de tracer un parcours historique assez exhaustif des étapes de la Révolution de Saint-Domingue et du déroulement des événements qui ont porté à la première abolition de l'esclavage en France $(1792-1794)^{14}$.

L'insurrection des esclaves de Saint-Domingue, op. cit., p. 15-27; Florence Gauthier (éd.), Périssent les colonies, op. cit., p. 23-41.

12. Jean-Daniel Piquet «Le Créole Patriote, apôtre de l'insurrection de St-Domingue», loc. cit., p. 519.

13. Les auteurs utilisent principalement les sources conservées dans les Archives Nationales de Paris, Sophie Piollet, Nathalie Piquionne, Delphine Roux, «Milscent créole historien de la Révolution de Saint-Domingue 1790-1794", dans Florence Gauthier (éd.), Périssent les colonies, op. cit., p. $23-42$.

14. En particulier à l'arrivée dans la colonie du décret du 24 mars - 4 avril 1792 jusqu'à l'abolition de l'esclavage ratifiée par le décret du 16 pluviôse an II : BHVP, 109109, Le Créole patriote, bulletin de Milscent-Créole, journal du soir, Paris, MilscentCréole, n 35, 1792-an II (1794), n 25 (jeudi matin, 4 octobre 1792), p. 99-104; AN, AD/ $\mathrm{XXa} / 176$, vol. 4, Le Créole patriote, bulletin de Milscent-Créole, journal du soir, $\mathrm{n}^{\circ}$ 42, 16 pluviôse an II (4 février 1794). 
Le Créole patriote est publié du 21 septembre 1792 au 19 ventôse an II (9 mars 1794) $)^{15}$. Il est difficile d'interpréter la source, à cause du langage révolutionnaire, mais on peut lire la section «Colonies» du journal sous deux angles: d'une part, Milscent fournit une sorte de chronique des événements, et d'autre part, il nous livre des commentaires subjectifs où émerge sa pensée abolitionniste. Sa pensée en particulier subit une évolution à partir de la fin du 1792. Les épîtres contenues dans le journal nous ont mis devant la problématique d'un premier niveau de médiation entre nous et l'objet historique. Le double parcours que nous avons voulu suivre est le suivant: d'un côté, une tentative de reconstruction historique à partir du regard de Milscent et de ses interlocuteurs et, d'un autre côté, une analyse de sa pensée abolitionniste en relation avec son profil biographique. Bien entendu, cette division est simpliste étant donné que les deux dimensions s'entrecroisent continuellement. Le parcours même de la vie de Milscent s'inscrit dans la période historique décrite.

Dans la pensée de Milscent, on constate une évolution dans un sens plus explicitement abolitionniste pendant les deux années prises en considération. Premièrement, Milscent soutient un projet d'abolition graduelle de l'esclavage dans son Essai sur l'amélioration du sort des esclaves, inclus dans son ouvrage Du régime colonial. À l'esclave on doit accorder « une sorte de propriété réelle ${ }^{16}$ », pour qu'il s’affranchisse et se transforme en un propriétaire, donc de "propriété » qu'il était, l'esclave doive devenir un «propriétaire». Lesclave, alors, change de statut juridique et il accède au statut de personne juridique, étant donné qu'avant, le statut juridique de l'esclave dans les colonies françaises se référait au Code noir (1685) qui contenait la contradiction entre les binômes: «esclave-personne» et «esclave-non personne». Ainsi donc, d'abord l'abolition graduelle aurait rendu l'esclave libre en tant que propriétaire de biens et dans un deuxième temps, le proprium l'aurait transformé en un homme juridiquement libre.

15. BHVP, 109109, Le Créole patriote, bulletin de Milscent-Créole, journal du soir, du n ${ }^{\circ} 1$ (vendredi soir, 21 septembre 1792); AN, AD/XXa/176, vol. 4, Le Créole patriote, bulletin de Milscent-Créole, journal du soir, $n^{\circ}$ 74, 19 ventôse an II (9 mars 1794).

16. BHVP, 955317 ; B.N. LK-9-193: Claude-Louis Milscent de Mussé, «Essai sur l'amélioration du sort des esclaves », dans Du régime colonial, 18 décembre 1791, Paris, Imprimerie du Cercle Social, 1792, p. 31. 
Deuxièmement, on constate une évolution dans un sens plus explicitement abolitionniste. On retrouve un témoignage de cette évolution à partir de la publication et défense de la Lettre originale des chefs Nègres révoltés, à l'assemblée générale, aux commissaires nationaux et aux citoyens de la partie Française de Saint-Domingue, du mois de Juillet 1792 - rédigée par les chefs des esclaves Biassou, JeanFrançois et Belair et envoyée à la Convention ${ }^{17}$. On peut déduire de ce texte que les esclaves imitaient les compatriotes français dans leur lutte contre l'oppression et que leur lutte avait trouvé fondement et inspiration dans la révolution éclatée dans la mère patrie. La lettre démontre donc que les esclaves avaient une parfaite connaissance des événements métropolitains. Un autre témoignage de l'adhésion aux événements de la Révolution française émerge de la section «Colonies » du Créole patriote du 20 décembre 1792, dans laquelle on trouve la description des réjouissances de la ville du Cap Français à l'arrivée de la nouvelle de la journée du 10 août $1792^{18}$. Dans la direction indiquée par l'historien Anne Pérotin on peut parler alors - à propos du réseau métropole-colonie - en termes de mimesis ${ }^{19}$. Milscent compare réellement la journée du 19 septembre à Saint-Domingue à la journée de la Prise des Tuileries, comme si la barrière temporelle de deux mois temps de voyage des nouvelles - pouvait être symboliquement dépassée. La lettre «des chefs Nègres » démontre comment la revendication des esclaves repose sur la Déclaration des droits de l'homme et du citoyen (26 août 1789 ) -elle se réfère en particulier à l'art. 2 : «Le but de toute association politique est la conservation des droits naturels et imprescriptibles de l'homme. Ces droits sont la liberté, la propriété, la sûreté et la résistance à l'oppression ${ }^{20}$.» Les esclaves détenaient donc le droit de rébellion au nom de la liberté à cause de la contradiction entre l'universalisme des principes et le particularisme législatif, qui

17. La lettre est publiée dans la section «Colonies $n^{\circ} 4$ " en BHVP, 109110, Le Créole patriote, bulletin de Milscent-Créole, journal du soir, $\mathrm{n}^{\circ} 282$ (samedi soir, 9 février 1793), p. 1148; N. Piquionne, «Lettre de Jean-François, Biassou et Belair », Annales historiques de la Révolution française, $\mathrm{n}^{\circ}$ 311, 1998, p. 132-139.

18. BHVP, 109110, Le Créole patriote, bulletin de Milscent-Créole, journal du soir, $\mathrm{n}^{\circ} 180$ (jeudi matin, 20 décembre 1792).

19. Anne Pérotin-Dumon, «Les Jacobins des Antilles ou l'esprit de liberté dans les îles-du-Vent", Revue d'histoire moderne et contemporaine, 35, 1988, p. 275-304.

20. Nathalie Piquionne, «Lettre de Jean-François, Biassou et Belair », op. cit., p. 133 . 
de fait excluait quelques sujets du système juridique. Mais en même temps les esclaves restaient une propriété et le droit de propriété restait un droit inaliénable, de sorte que les colons pouvaient revendiquer leur droit esclavagiste et accorder l'affranchissement seulement s'ils avaient été indemnisés: «la société coloniale: mieux qu’une hiérarchie, une ontologie: en haut, le blanc - l'être au sens plein du terme -, en bas, le nègre, sans personnalité juridique, un meuble ${ }^{21}$ ».

La contribution abolitionniste de Milscent et le rapport entre la métropole et sa colonie émergent dans les lettres de «Hivvo à son ami Saii ${ }^{22}$ ", une invention littéraire de l'auteur. Les personnages ne sont pas réels, mais bien que caricaturaux, ils incarnent tout de même des situations réelles. Les lettres rendent manifeste que la période révolutionnaire n'a pas été seulement un ensemble d'événements mais aussi un espace de prolifération des écrits. Au delà du contenu éminemment politique des épîtres, l'aspect littéraire et dialogique nous présente une «fresque » intéressante de ce que put signifier pour un «sauvage»c'est ainsi que Hivvo se définit de manière ironique, à la fin du XVIII ${ }^{\mathrm{e}}$ siècle -, de nouer le contact avec les habitants de la métropole, de devoir affronter l'altérité de leur mentalité et de leur coutume, et enfin de devoir comprendre le temps de la Révolution en France. Par exemple, dans la cinquième lettre de Hivvo à Saï ${ }^{23}$, le journaliste créole se montre solidaire avec les esclaves en soutenant la légitimité du droit de résistance. La liberté est entendue soit comme résistance de l'esclave contre le maître dans l'espace colonial, soit, dans une perspective

21. Aimé Césaire, op. cit., p. 33.

22. Les lettres de Hivvo à son ami Saï ont été publiées dans BHVP, 10911o, Le Créole patriote, bulletin de Milscent-Créole, journal du soir, $\mathrm{n}^{\circ} 289$ (mercredi matin, 13 février 1793); $n^{\circ} 291$ (jeudi matin, 14 février 1793); n 295 (samedi matin, 16 février 1793); $\mathrm{n}^{\circ} 297$ (dimanche matin, 17 février 1793); nº 301 (mardi matin, 19 février 1793); $\mathrm{n}^{\circ} 303$ (mercredi matin, 20 février 1793); nº 305 ( jeudi matin, 20 février 1793). Dans la première lettre, on trouve que le rapport épistolaire commença en 1778 et que Milscent publia seulement une partie des lettres. On peut déduire à partir du contenu qu'il s'agit de l'année 1793 «Lettre de Hivvo, sauvage des Apalaches, à son ami Saï, aussi sauvage du même lieu, mais actuellement au Cap-Français. Paris, le 22 janvier [...] l'an 276 de l'arrivée de Fernand Cortez à l'Amérique. (1) n $n^{\circ} 1$ ». Une note précise: «(1) Ces lettres, commencées en 1778, ne sont pas encore imprimées: je ne donnerai dans ce journal que celles qui seront écrites depuis l'arrivée de Hivvo à Paris pour la seconde fois ", cité dans la section Variétés BHVP, 109110, Le Créole patriote, bulletin de Milscent-Créole, journal du soir, $n^{\circ} 289$ (mercredi matin, 13 février 1793), p. 1175.

23. "Lettre de Hivvo à Saï, $\mathrm{n}^{\circ} 5$ ", BHVP, 109110, Le Créole patriote, bulletin de Milscent-Créole, journal du soir, nº 301 (mardi matin, 19 février 1793), p. 1223. 
globale, comme la révolution d'un groupe d'esclaves qui légitimement la lutte pour l'autonomie contre la mère patrie. Notamment, il y a une transformation d'une lutte antiesclavagiste en une lutte anticolonialiste. On a cherché à mettre en relief la différence entre le concept de «droit de résistance à l'oppression » qui remonte au droit naturel et le concept de «liberté » qui remonte au désir de l'esclave pour la liberté, sans être nécessairement lié à ce droit.

On peut en conclure que le concept de citoyenneté soutenu par la Déclaration des droits avait des frontières géographiques, économiques et sociales bien précises et que dans l'univers colonial, la portée universaliste de la Révolution n'était pas arrivée là où elle est arrivée seulement grâce à la contribution de personnages comme Milscent. Les limites de l'universalisme furent dépassées par l'effort des esclaves vers la liberté qui influença énormément le processus qui conduisit à l'abolition de l'esclavage le 16 pluviôse an II. Milscent - en croyant fermement dans le droit de résistance à l'oppression et dans le droit inaliénable de la liberté - se solidarisait avec les esclaves qui rendaient réellement vivants les principes de la Déclaration des droits de l'homme et du citoyen.

Même si l'utilisation du journal Le Créole patriote comme source n’a pas été d'interprétation facile pour la compréhension du déroulement de la Révolution de Saint-Domingue (1792-1794), on a essayé de tracer un double parcours avec le soutien des études précédentes. Après, on a cherché à élargir et confronter les sujets traités dans le journal avec ceux traités par les études les plus importantes sur la Révolution de Saint-Domingue, en prenant en considération que la majorité des faits historiques racontés était le fruit des rapports épistolaires entre Milscent et ses interlocuteurs coloniaux : les hommes libres de couleur et les esclaves ${ }^{24}$.

Du point de vue de la reconstruction historique, la nouvelle de la réception dans la colonie du décret du 24 mars-4 avril 1792 a été déter-

24. Yves Bénot, La Révolution française et la fin des colonies, op. cit., p. 54, 83, 84, 120, 166, 167, 168, 172, 175, 185, 196, 217 et 241; Jean-Daniel Piquet, «Le Créole Patriote, apôtre de l'insurrection de St-Domingue », op. cit.; Florence Gauthier, "Comment la nouvelle de l'insurrection des esclaves de Saint-Domingue fut-elle reçue en France?», op. cit.; Sophie Piollet, Nathalie Piquionne, Delphine Roux, «Milscent créole historien de la Révolution de Saint-Domingue 1790-1794", op. cit. Sur Milscent, voir aussi David Patrick Geggus, Haitian Revolutionary Studies, Bloomington, Indiana University Press, 2002, p. 71 et 76 . 
minante puisqu'elle a montré comment, à l'intérieur de la complexe société coloniale, les colons blancs avaient réagi à l'élargissement des droits en faveur des hommes libres de couleur. Dans l'«Extrait d'une lettre du Cap, en date 3 août 1792 ", on trouve que le décret a été accepté favorablement non seulement par les nouveaux citoyens, les hommes libres de couleur, mais aussi par les colons. Par contre, dans d'autres numéros du journal, on a trouvé que les hommes libres de couleur étaient encore des victimes des abus des colons ${ }^{25}$. Le "préjugé de couleur ${ }^{26} »$ n'avait pas encore décliné dans l'univers colonial. En réalité, il n’avait pas décliné non plus dans l'univers métropolitain. De même, dans les premières années révolutionnaires, on assiste à une aggravation du phénomène du préjugé de couleur qui va exactement dans la direction contraire de celle des principes de la Déclaration des droits $(1789)^{27}$. On a relevé cette tendance dans la sixième des «Lettres de Hivvo à Saï ${ }^{28}$ », dans laquelle Hivvo est défini comme «étranger » pour sa couleur de peau et pour l'habillement différent qu'il porte. Après l'éclatement de la Révolution française, à propos des colons et de la complexité de la hiérarchie coloniale, la mère patrie n’avait pas l'intention d'interférer avec l'autonomie locale coloniale. En substance, comme Yvan Debbasch le soutient, la métropole cherche à maintenir la compétence législative à propos du monopole commercial de la colonie, donc de son régime extérieur, l'exclusif colonial, mais elle ne veut pas interférer avec le régime intérieur ${ }^{29}$. Lautonomie locale devait avoir comme objectif le maintien d'une condition de statu quo

25. Bernard Gainot démontre la diversité des positions politiques à la suite de la réception de la nouvelle du décret du 4 avril 1792, alors que les colons blancs tentent de rétablir l'ordre esclavagiste dans la ville du Cap (Bernard Gainot, «Le général Laveaux, gouverneur de Saint Domingue, député néo-jacobin », Annales historiques de la Révolution française, $\mathrm{n}^{\circ} 278$, 1989, p. 437).

26. Florence Gauthier, Laristocratie de l'épiderme. Le combat de la Société des Citoyens de Couleur 1789-1791, Paris, CNRS Éditions, 2007, p. 226-230. Sur le préjugé de couleur voir aussi Fédéric Régent, La France et ses esclaves. De la colonisation aux abolitions (1620-1848), Paris, Grasset, 2007, p. 194-195.

27. Voir «Vers la constitutionnalisation de l'esclavage et du préjugé de couleur par la Constituante Mars 1790-Mai 1791 », dans Florence Gauthier, Laristocratie de l'épiderme, op. cit, p. 259.

28. «Lettre de Hivvo, à son ami Saï, nº 6 ", dans BHVP, 109110, n 303 (mercredi matin, 20 février 1793), p. 1231.

29. Yvan Debbasch, Couleur et liberté, le jeu du critère ethnique dans un ordre juridique esclavagiste. I: Laffranchi dans les possessions français de la Caraïbe ${ }_{116} 5_{5-}^{-}$ 1833), Paris, Dalloz, 1967, p. 188. 
ante révolution. C'est dans ce contexte que le décret de constitutionnalisation de l'esclavage fut mis en œuvre par l'assemblée coloniale législative de la partie française de l'île. Milscent, au contraire, après avoir rapporté cette nouvelle, se demandait: «Est-il bien vrai que Saint-Domingue ne puisse exister sans le maintien de l'esclavage ${ }^{30}$ ? "

Milscent était contre la politique esclavagiste des grands colons. Dans la section "Colonies $n^{\circ} 8$ ", les petits blancs avaient été définis comme les vrais sans-culottes de l'île. Ils étaient toujours solidaires avec les libres de couleur et pas hostiles à l'abolition de l'esclavage ${ }^{31}$. Lutilisation de l'expression «sans-culottes» pour la définition des petits blancs est une démonstration de la connexion, au niveau linguistique, entre les deux révolutions et de la manière dont le langage révolutionnaire avait réussi à pénétrer en profondeur dans la dimension locale de la colonie. À l'appui de la thèse des implications globales de la question abolitionniste, a émergé la preuve de la coalition entre les colons indépendantistes, les Léopardins, et la puissance coloniale anglaise, avant la déclaration de guerre de la Gironde à l'Angleterre, le $1^{\text {er }}$ février 1793. On trouve notamment le témoignage d'un projet indépendantiste du lobby colonial esclavagiste soutenu par le gouvernement britannique de William Pitt ${ }^{32}$. Cependant, l'Angleterre intervint militairement, en débarquant sur l'île de Saint-Domingue le 19 septembre 1793, après la proclamation de la première abolition de l'esclavage menée par le commissaire civil Léger Félicité-Sonthonax. Encore, à propos des implications internationales, on retrouve le soutien espagnol aux esclaves rebelles dans une lettre datée du 3 août 1792, dans laquelle les chefs Jean-François et Biassou avaient envoyé à la fin de juillet une pétition à l'assemblée coloniale pour proposer une abolition ${ }^{33}$.

30. BHVP, 109109, Le Créole patriote, bulletin de Milscent-Créole, journal du soir, $n^{\circ} 136$ (mercredi soir, 28 novembre 1792).

31. BHVP, 109110, Le Créole patriote, bulletin de Milscent-Créole, journal du soir, $\mathrm{n}^{\circ} 290$ (mercredi soir, 13 février 1793), p. 1180.

32. Florence Gauthier, Triomphe et mort du droit naturel en Révolution, op. cit., p. 208-212.

33. Sur le soutien de L'Espagne aux esclaves voir BHVP, 109109, Le Créole patriote, bulletin de Milscent-Créole, journal du soir, $\mathrm{n}^{\circ} 44$ (samedi soir, 13 octobre 1792). Sur le projet indépendantiste soutenu par l'Angleterre voir BHVP, 109109, Le Créole patriote, bulletin de Milscent-Créole, journal du soir, $\mathrm{n}^{\circ} 69$ (vendredi matin, 26 octobre 1792). 
De l'autre parcours, lié au profil biographique de Milscent et à sa pensée antiesclavagiste, il ressort qu'un petit colon pouvait être antiesclavagiste, et ce, pas seulement en théorie. L'évolution de la pensée antiesclavagiste de Milscent et les propositions innovatrices qu'il porta au cour du débat public en France doivent être replacées au sein des changements en cours. La pensée abolitionniste de Milscent et sa biographie sont intimement tressées et paradoxalement le journaliste créole, ayant aussi été un des partisans du débat qui mena à l'abolition du 4 février 1794-16 pluviôse an II, trouva la mort sous la Convention Montagnarde, accusé d'avoir tué des esclaves et d'avoir participé à l'assemblée coloniale dans la ville du Cap Français.

Laccusation, injuste, d'avoir politiquement soutenu Brissot - qui lui coûta à lui et à son journal l'expulsion du club des Jacobins le 28 décembre 1793 - fut causée par la campagne du parti colonial esclavagiste, à la tête duquel on retrouve les colons patriotes Page et Brulley $^{34}$. La démonstration de l'accusation infondée réside dans Le Créole patriote, dans lequel on constate que Milscent avait écrit contre et non pas a faveur de Brissot ${ }^{35}$. Le fondateur de la Société des Amis des Noirs fut accusé par Milscent de ne pas être vraiment antiesclavagiste et de vouloir défendre le statu quo colonial à Saint-Domingue. Par conséquent, on peut conclure que Milscent ne reconnaissait pas la contribution abolitionniste du chef de la Gironde. L'accusation d'être en faveur de Brissot est probablement liée à la collaboration de quelques mois (21 février 1793-5 avril 1793) avec le journal d'orientation girondine, le Bulletin des Amis de la Vérité. Le journaliste créole fut forcé d'accepter cette nouvelle collaboration éditoriale à cause des difficultés

34. AN, AD/XXa/176, vol. 3, Le Créole patriote, bulletin de Milscent-Créole, journal du soir, $\mathrm{n}^{\circ} 7$ du 11 nivôse an II (31 décembre 1793). La campagne diffamatoire de Page et Brulley fut découverte et ils furent arrêtés le 16 ventôse an II (6 mars 1794). Sur le démantèlement du réseau colon infiltré en France, voir Claire Blondet, "Quand les "terroristes" font le procès du colonialisme esclavagiste, les thermidoriens organisent son oubli ", dans Florence Gauthier (éd.), Périssent les colonies, op. cit., p. 48.; Florence Gauthier, Triomphe et mort du droit naturel en Révolution, op. cit., p. 225-230.

35. «Depuis la fondation de la République, le parti de Barnave est définitivement vaincu. Que fait alors Brissot? Il divise la Convention pour se trouver un nouvel adversaire et faute d'avoir pu en trouver, colonialiste et esclavagiste, il défend le statu quo à Saint-Domingue en envoyant des troupes combattre les esclaves? ", dans BHVP, 109110, Le Créole patriote, bulletin de Milscent-Créole, journal du soir, $\mathrm{n}^{\circ} 295$ (samedi matin, 16 février 1793), p. 1199. 
financières qui le conduisirent à arrêter la publication de son journal. Avec les numéros du journal actuellement disponibles, il n’a pas été possible de reconstruire exactement quand reprit la publication du Créole patriote, puisque beaucoup de numéros manquent dans la période chronologique comprise entre le 5 avril 1793 et le 15 octobre 1793. La bataille de Milscent dut s'arrêter définitivement le 9 mars 1794. Le décret du 19 ventôse an II (9 mars 1794), qui décrétait l'arrêt des colons ayant appartenu à une assemblée coloniale ou au club Massiac, englobait Milscent, qui fut condamné à la peine de mort le 7 prairial an II (26 mai 1794). À partir de l'automne 1793, Milscent avait noté que «Laffaire des colonies était tellement embrouille, et [...] falsifiée, qu’il n'était presque plus possible d'y asseoir un jugement ${ }^{36}{ }^{\prime}$. Lattaque que Milscent subit du montagnard Collot-d'Herbois se référait spécifiquement au rôle qu'il avait endossé dans l'île comme capitaine de milice chargé de réprimer les différents mouvements des esclaves marrons: «Je prie le président de demander à Milscent si, dans la colonie, il n'a pas fait la chasse aux nègres dans les bois, et s'il n'en a pas tué un grand nombre ${ }^{37}$. $\gg$ De ces mots, on pourrait déduire qu'il existe une contradiction entre la pensée antiesclavagiste de Milscent et le rôle qu'il endossa comme capitaine de milice, mais en réalité c'est un anachronisme. Pendant l'analyse entière on a pu constater que la pensée antiesclavagiste de Milscent arriva à pleine maturation au cours de l'année 1792 et que sa charge comme lieutenant datait d'une date antérieure $: 1774^{38}$. On ne sait pas si l'évolution de la pensée de Milscent avait été motivée par une réflexion intérieure ou par la contingence révolutionnaire puisque le mot révolution signifie justement, entre autres, que le rythme à l'improviste change.

L'abolitionnisme de Milscent apparait fortement dans les « Lettres de Hivvo à son ami Saï» où Milscent-Hivvo s'était identifié avec la figure de l'«esclave» et y abordait la question de la révolte des esclaves. À ce propos, Milscent évoque la révolte des esclaves en se référant aux

36. AN, AD/XXa/176, vol. 3, Le Créole patriote, bulletin de Milscent-Créole, journal du soir, $\mathrm{n}^{\circ} 84$ (17 octobre 1793).

37. AN, AD/XXa/176, vol. 3, Le Créole patriote, bulletin de Milscent-Créole, journal du soir, $\mathrm{n}^{\circ} 7$ du 11 nivôse an II (31 décembre 1793).

38. BHVP 961 248; B.N. 8-LK12-362: Claude-Louis Milscent de Mussé, Sur les troubles de Saint-Domingue, 31 octobre 1791, Paris, Imprimerie du Patriote François, p. 10. 
principes du droit naturel et à l'application des droits universels de la Révolution française. Du point de vue du sauvage des Appalaches, l'esclave se rebelle en tant qu'il a reconnu une usurpation sur le plan du droit. Donc l'esclave semble comprendre la contradiction entre principes constitutionnels et action politique. En réalité, comme Carolyn E. Fick le soutient, il n'est pas dit que l'esclave ait connu cette contradiction ni que sa lutte antiesclavagiste se soit appuyée sur cette antinomie $^{39}$. À propos du droit à l'insurrection de l'esclave et au concept de liberté comme résistance à l'oppression et comme réalisation de la justice dans l'égalité, la révolte des esclaves de SaintDomingue semble naître plutôt du désir de liberté. En effet, la révolte des esclaves n'était pas ancrée sur le droit de rébellion, basé sur le droit naturel comme théorisation euro-centrique, mais était plutôt le fruit de l'aspiration de l'homme esclave à la libertét ${ }^{4}$.

Enfin, on peut soutenir que Milscent avait compris la portée historique de la révolte des esclaves et qu'il avait compris les limites mêmes de la Révolution française à propos de l'enjeu «politique des principes » et «politique des intérêts». En croyant fermement dans le droit de résistance à l'oppression et dans le droit inaliénable à la liberté, Milscent se solidarisait avec les esclaves, qui incarnaient pleinement les principes de la Déclaration des droits de l'homme et du citoyen.

39. Carolyn Fick, The Making of Haiti: The Saint Domingue Revolution from below, Knoxville, The University of Tennessee Press, 1990.

40. Ugo Maria Olivieri, «Introduzione », dans Étienne de la Boétie, Discorso sulla servitù volontaria, Turin, La Rosa, 1995, p. xvii. Une partie de l'historiographie soutient que la révolte des esclaves doit être interprétée comme un processus identitaire autonome ou qu'il serait mieux de parler de l'origine africaine de l'insurrection de 1791. Pour le premier aspect, voir Carolyn Fick, «La révolution de Saint-Domingue. De l'insurrection du 22 août 1791 à la formation de l'État haïtien », traduit par M. Villard, dans Laënnec Hurbon (éd.), L'insurrection des esclaves de Saint-Domingue, op. cit., p. 55-75; pour l'origine africaine du droit à l'insurrection, voir Robin Law, « La cérémonie du Bois Caïman et le "pacte de sang" dahoméen ", traduit par M. Villard, dans Laënnec Hurbon (éd.), L'insurrection des esclaves de Saint-Domingue, op. cit., p. 133 . 